\title{
Spotlight: Automated Confidence-based User Guidance for Increasing Efficiency in Interactive 3D Image Segmentation
}

\author{
Andrew Top ${ }^{1}$, Ghassan Hamarneh ${ }^{1}$ and Rafeef Abugharbieh ${ }^{2}$ \\ 1 Medical Image Analysis Lab, Simon Fraser University, \\ \{atop, hamarneh\}@cs.sfu.ca \\ 2 Biomedical Signal and Image Computing Lab, University of British Columbia, \\ rafeef@ece.ubc.ca
}

\begin{abstract}
We present Spotlight, an automated user guidance technique for improving quality and efficiency of interactive segmentation tasks. Spotlight augments interactive segmentation algorithms by automatically highlighting areas in need of attention to the user during the interaction phase. We employ a 3D Livewire algorithm as our base segmentation method where the user quickly provides a minimal initial contour seeding. The quality of the initial segmentation is then evaluated based on three different metrics that probe the contour edge strength, contour stability and object connectivity. The result of this evaluation is fed into a novel algorithm that autonomously suggests regions that require user intervention. Essentially, Spotlight flags potentially problematic image regions in a prioritized fashion based on an optimization process for improving the final 3D segmentation. We present a variety of qualitative and quantitative examples demonstrating Spotlight's intuitive use and proven utility in reducing user input by increasing automation.
\end{abstract}

\section{Introduction}

Manual segmentation in 3D medical images is extremely time consuming and tedious. Despite the huge effort often involved, manual segmentation typically suffers from high operator variability and less than ideal results due to user fatigue [11]. Numerous automated algorithms have been developed to aid in image segmentation. Unfortunately, current state-of-theart fully-automatic algorithms still have difficulty segmenting highly variable shapes such as anatomical structures, hence requiring considerable training resources as well as initialization and fine tuning of unintuitive parameters. Automatic methods cannot guarantee perfect segmentations since the user is not directly involved in the segmentation process. It can thus be rather difficult to correct errors of automatic segmentation, necessitating a final quality assurance pass using some separate interactive segmentation editing tool $[8,6]$. 
Semi-automatic methods have been long introduced [11], where the user is factored in to play a larger role in guiding the segmentation process and in correcting errors as they occur. For example, graph cuts [3] and random walker algorithms $[5,7]$ can be used for interactive segmentation by specifying object and background seeds. Alternative approaches such as Livewire [2] detect contours in 2D by finding the minimal cost path through user specified seedpoints in the image. Livewire has been shown to be highly reproducible [2], and since the user can see the path forming in real-time, errors can be corrected as they appear. Malmberg et al. [10] attempted to extend Livewire to 3D by removing the restriction of Livewire contours to planes but, unfortunately, the interface used to create the non-planar user-specified contours requires special haptic hardware. Armstrong et al. [1] suggested a 3D analog of a 2D Livewire, namely a "Live Surface", which can be localized in real-time through an optimized graph cuts algorithm. The more recent turtle map 3D Livewire algorithm [12], or T-LW for short, accepts a sparse set of user-specified planar contours as input, which it automatically combines to seed unvisited planes and subsequently generate a new dense set of 3D contours.

A common problem among current interactive segmentation algorithms is that while they generally produce better results as the user provides more input, it is typically not clear to the user what extra input would most improve the segmentation and where additional intervention would be optimal. To address this optimal input problem, we propose an approach that draws the user's attention to low-confidence regions in intermediate segmentations, prodding him/her to provide more information. Effectively, we are automating the process of choosing where additional input should be added, relaxing the burden on the user. In analogy to how theatres use spotlights to focus the audience's attention to an interesting area on the stage, we name our technique 'Spotlight'.

\section{Method}

We build Spotlight on top of an enhanced version of the T-LW algorithm [12]. Although other base methods such as graph cuts and random walker exist and may be adopted (see the Conclusions section), we chose T-LW because it allows for the more natural interaction experience of contouring within 2D slice planes. Users specify seeds along the boundary of the object, enforcing the segmentation to go through it. The reader is referred to the T-LW paper [12] for a detailed explanation of the algorithm. In

\footnotetext{
${ }^{1}$ A software implementation can be found at http://www.turtleseg.org
} 
this work, the T-LW user interaction mechanism remains the same but with the addition of a much needed "suggest plane" feature, which invokes Spotlight to locate a 2D LW contouring plane chosen optimally in regions of maximal segmentation ambiguity, which in turn improves accuracy and speeds up the interactive segmentation process.

In order to enable optimal plane suggestions, which can naturally be in any oblique orientation, we first extend the T-LW algorithm to support user contour specification in arbitrary views as opposed to only the three orthogonal fixed views (section 2.1). We then arm T-LW with our proposed automatic plane suggestion technique which consists of two components; segmentation evaluation (section 2.2) and contour plane suggestion (section 2.3). Three evaluation criteria based on different metrics create a set of objects called Spotlight Attractors (SAs), which indicate suspected poor segmentation quality regions. Each Spotlight Attractor is a triplet $(\boldsymbol{p}, \boldsymbol{n}, s)$, where $\boldsymbol{p}$ and $s \in[0,1]$ are the SA's 3D position and strength, respectively, and $\boldsymbol{n}$ is a unit-length direction vector that influences the orientation of the suggested plane. Subsequently, the plane suggestion component determines the 'optimal' high priority plane that passes through the vicinity of the SA positions and conforms to the normals of as many strong SAs as possible.

In summary, a user provides a sparse set of initial contours of arbitrary orientation, and then proceeds to iterate between applying the turtle map algorithm to obtain a 3D Livewire segmentation and examining what Spotlight has flagged as a problematic region for potential intervention. At no point is the user locked to any specific sequence of events, rather the method allows for total freedom in choosing the image planes to be segmented at all times.

\subsection{Extending T-LW to Oblique Slices}

The T-LW algorithm [12] automatically generates Livewire contours for an unvisited plane $\mathcal{P}_{m}$ based on the existing sparse set of interactivelyseeded $2 \mathrm{D}$ contours. A plane $\mathcal{P}_{m}$ will contain a turtle map, which is a set of line segments resulting from intersecting $\mathcal{P}_{m}$ with the user-generated contours in other planes not parallel to $\mathcal{P}_{m}$ (Fig. 1).

The turtle map encodes the position and ordering of automatically generated Livewire seedpoints in $\mathcal{P}_{m}$. In order to support oblique contouring slices, we develop a continuous analytical representation of the turtle map instead of the discrete binary bitmap of [12] that is restricted to the pixels in the standard three orthogonal views. We achieve this by taking the line segments produced by intersecting each user contour with 


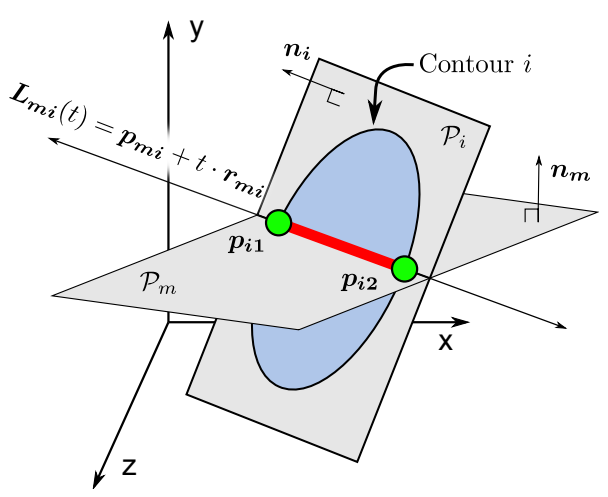

Fig. 1. Computing the turtle map line segments: For contour $i$ (shown encapsulating the blue region in the contour plane $\left.\mathcal{P}_{i}\right), \boldsymbol{L}_{\boldsymbol{m i}}(t)$, with real parameter $t$, is the parameterized line formed by intersecting the unvisited plane $\mathcal{P}_{m}$ with $\mathcal{P}_{i} . \boldsymbol{p}_{\boldsymbol{m} i}$ is a point on $\boldsymbol{L}_{\boldsymbol{m} i}$ and $\boldsymbol{r}_{\boldsymbol{m} i}$ is the direction of $\boldsymbol{L}_{\boldsymbol{m} i \boldsymbol{i}}$. The points $\boldsymbol{p}_{\boldsymbol{i 1}}$ and $\boldsymbol{p}_{\boldsymbol{i} \mathbf{2}}$ are where $\boldsymbol{L}_{\boldsymbol{m} \boldsymbol{i}}$ intersects contour $i$, and form a (red) turtle map line segment on the turtle map (Fig. 2).

$\mathcal{P}_{m}$ (the red line segment in Fig. 1), and then forming a graph where the vertices are the line segment end points (green) and intersection points (pink), as seen in Fig. 2. The endpoints of the graph represent Livewire seeds on $\mathcal{P}_{m}$. Note that although $\mathcal{P}_{m}$ is unvisited by the user, the seeds on $\mathcal{P}_{m}$ belong to segmentations already confirmed by the user. To allow for fully automatic Livewire contouring in the unseen $\mathcal{P}_{m}$, the seedpoints are ordered by choosing an arbitrary initial vertex and then traversing the graph. The next vertex chosen in our graph traversal strategy is the one whose edge forms the smallest oriented angle with the previous vertex (Fig. 2). The automatically generated and ordered seeds on the unvisited plane $\mathcal{P}_{m}$ are then used to fully automatically livewire the object in $\mathcal{P}_{m}$. A final 3D segmentation is obtained by choosing a dense set of planes parallel to $\mathcal{P}_{m}$, and then applying the procedure above to each of them.

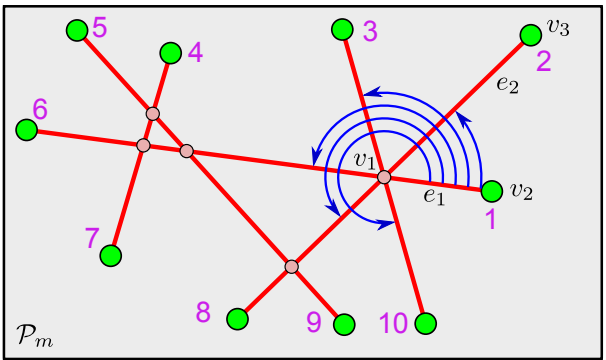

\subsection{Segmentation Assessment}

Following the automatic construction of a 3D segmentation, three confidence criteria (i-iii) are evaluated for all automatically-segmented slices.

(i) Contour Edge Strength Due to potential lack of seedpoints in unvisited slices, the automatically generated contour may incorrectly cut
Fig. 2. Line segment end points (Fig. 1) become unordered Livewire seeds. The seeds are ordered as in this example: at intersection vertex $v_{1}$ arriving from $e_{1}$, the next edge is that which forms the smallest counter clockwise angle with $e_{1}$, in this case, $e_{2}$. Following $e_{2}$ gives the vertex after $v_{2}$ as $v_{3}$. The purple numbers show the ordering from repeating this procedure. 
through homogeneous (i.e. edge-free) regions of the object. At such locations, the Livewire local edge cost of the resulting contour (normalized by length) will be high. Hence, for each sample $j$ created by sampling the generated contour at equal arc length intervals, we obtain a position $\boldsymbol{p}_{\boldsymbol{j}}$ and a corresponding normal $\boldsymbol{n}_{\boldsymbol{j}}$ (normal to the contour and lying in the contour's plane), which are then used to generate a SA: $\left(\boldsymbol{p}_{\boldsymbol{j}}, \boldsymbol{n}_{\boldsymbol{j}}, f_{L W j}\right)$. Here, $f_{L W j}$ is the Livewire local edge cost at point $j$, based on a combined Canny edge, Laplacian of Gaussian, gradient magnitude and gradient direction terms [12].

(ii) Contour stability The Livewire algorithm calculates the globally minimal path between two points, but gives no evidence of other near-optimal paths. Inspired by the k-shortest paths problem [13], we detect such paths by perturbing single edges in the shortest path (Fig. 3) to produce a set $\mathcal{D}$ of perturbed paths. Our contour instability value is calculated, at each point $k$ on the contour, as the maximum Euclidean distance between the position $\boldsymbol{p}_{\boldsymbol{k}}$ of $k$ and each perturbed path, given by $d_{k}=\max _{R \in \mathcal{D}}\left(\min _{\boldsymbol{p} \in R}\left(\left|\boldsymbol{p}_{\boldsymbol{k}}-\boldsymbol{p}\right|_{2}\right)\right)$. We then create the SA as $\left(\boldsymbol{p}_{\boldsymbol{k}}, \boldsymbol{n}_{\boldsymbol{k}}, u\left(d_{k}\right)\right)$, where $\boldsymbol{p}_{\boldsymbol{k}}$ and $\boldsymbol{n}_{\boldsymbol{k}}$ are as before (in (i)) and $u(x)$ is a monotonically increasing function that assigns a strength $(\in[0,1])$ to the distance. In our implementation, $u(x)$ normalizes the distance with respect to the largest distance between points in the existing contours.

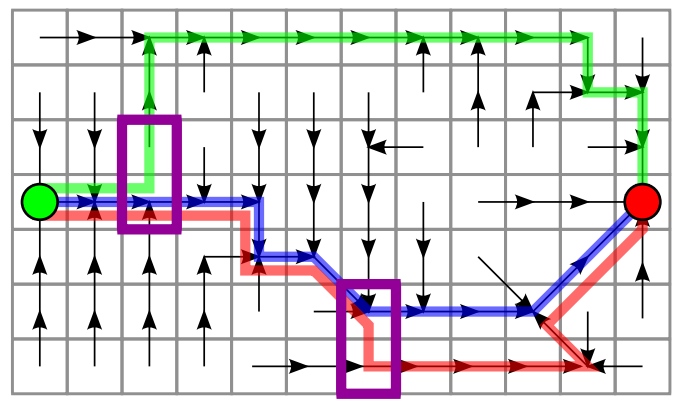

Fig. 3. Path perturbation example. New paths (green and red) formed through perturbations of the shortest path (blue). The path perturbation edges are outlined in purple. The thin black arrows represent the shortest path tree from the source (green dot) to the destination (red dot). Note how a local change can result in a substantially different path (green).

(iii) Turtle Map Connectivity This metric detects unvisited planes that lack the seeds necessary to reveal the cross section of the object. A turtle map containing non-intersecting line segments would provide only 2 seedpoints, typically resulting in a poor segmentation. For each nonintersecting line segment $h$ in an unvisited plane with normal $\boldsymbol{n}_{\boldsymbol{h}}$ within that plane, we sample points $i$ along $h$ at a resolution proportional to that of the source image. Finally, for each $i$, we generate a SA as $\left(\boldsymbol{p}_{\boldsymbol{i}}, \boldsymbol{n}_{\boldsymbol{h}}, 1\right)$. 


\subsection{Spotlight: Automated Slice Plane Suggestion}

In order to produce an optimal slice plane on which the user should focus his/her attention, we devise an objective function that assigns a cost to a plane given the set of generated SAs. At a high level, we derive a cost for the plane with respect to each SA, and sum these costs over all SAs. Given a plane $\mathcal{P}$ with normal $\boldsymbol{n}_{\mathcal{P}}$ and offset from origin $d_{\mathcal{P}}$, the cost of a suggested plane $\mathcal{P}$ is given by

$$
E(\mathcal{P})=\frac{\sum_{i \in S A} s_{i} E_{S A}(i, \mathcal{P})}{\sum_{i \in S A} s_{i}}
$$

where $s_{i}$ is the strength of attractor $i . E_{S A}(i, \mathcal{P})$ is the cost contribution associated with the $i$ th SA, which is low if the plane $\mathcal{P}$ is near and parallel to the SA's normal. Therefore, $E_{S A}$ is defined as

$$
E_{S A}(i, \mathcal{P})=q_{\mathcal{P}}(i)+\left(1-q_{\mathcal{P}}(i)\right) L\left(d\left(\boldsymbol{p}_{\boldsymbol{i}}, \mathcal{P}\right)\right) .
$$

Here, $q_{\mathcal{P}}(i)=\left(\boldsymbol{n}_{\boldsymbol{i}} \cdot \boldsymbol{n}_{\mathcal{P}}\right)^{2}$ describes how similar the plane's normal is to the Spotlight Attractor's, where the more perpendicular the normals are, the lower the value of $q_{\mathcal{P}}(i)$ and hence a value of $E_{S A}$ closer to $L\left(d\left(\boldsymbol{p}_{\boldsymbol{i}}, \mathcal{P}\right)\right)$. We set $d(\boldsymbol{p}, \mathcal{P})=\left|\boldsymbol{p} \cdot \boldsymbol{n}_{\mathcal{P}}-d_{\mathcal{P}}\right| . L:[0, \infty) \rightarrow[0,1)$ is a logistic shaped function used to reduce the influence of points far from $\mathcal{P}$ on the optimizer.

We use gradient descent to minimize $E$ with respect to $\mathcal{P}$. We iterate over multiple initialization planes, each passing through at least one SA, and pick the plane with the lowest minimum.

\section{Results}

\subsection{Implementation Details}

We have chosen the algorithm parameters empirically and kept them fixed during all the following experiments, showing that these fixed parameters are robust to a wide variety of image modalities and data. The main parameters used in the Spotlight-augmented segmentation process are those inherited from the T-LW algorithm. Spotlight-only parameters include the gradient descent parameters, as well as the relative weightings between the evaluation metrics. We keep the evaluation metric relative weightings equal. For the gradient descent parameters, we have found a good quality-performance balance at 40 re-initializions, with 80 steps performed each time. Increasing the gradient descent steps beyond these values increases the computation time only; the increase in suggestion plane quality is negligible. 


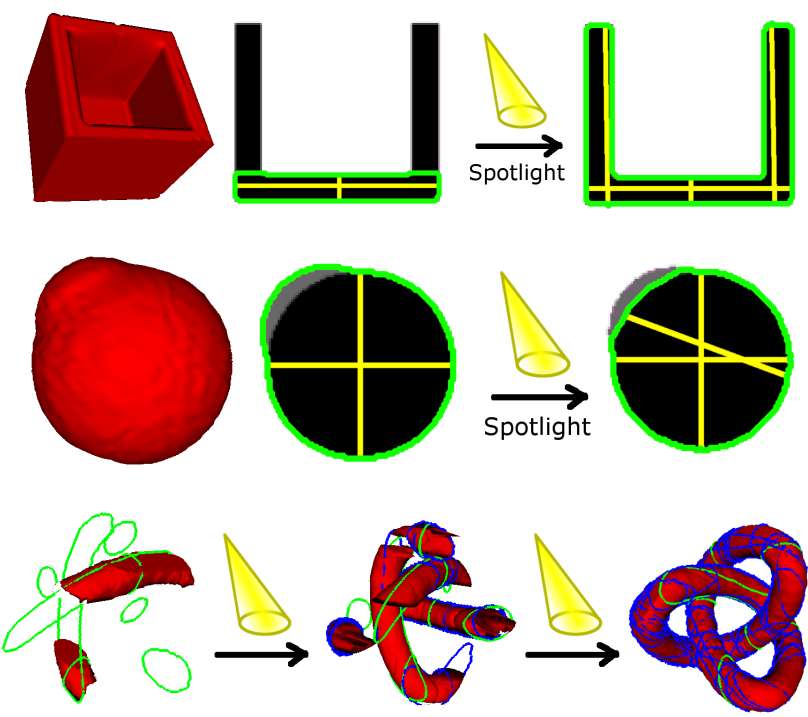

Fig. 4. Spotlight discovering segmentation mistakes. Note how in the top row the sides (vertical black bars) of the box is segmented properly, how the bulge is correctly excluded from the sphere in the second row, and Spotlight's proper handling of complicated non-spherical topology in the third row (see text for details).

\subsection{Synthetic tests}

Fig. 4 (row 1) illustrates an open box test example (left) where the initial turtle map cuts through the box walls (middle), creating high Livewire costs that are detected and fixed by Spotlight (right). Fig. 4 (row 2) shows a sphere with an unwanted bump caused by a strong nearby edge (left), creating high path stability costs (middle) causing Spotlight to suggest a slice plane that removes the ambiguity about where the Livewire path should follow (right). Fig. 4 (row 3) shows Spotlight guiding the segmentation of a knot, where starting with only 2 user-chosen slice planes (green) on the left, we follow numerous suggestions from Spotlight (blue) to fully segment the knot, seen on the right.

\subsection{Real Medical Data Tests}

Fig. 5 shows our tests on real medical images. In all examples, an initial set of contours (green) is provided by the user, who proceeds to contour Spotlight suggested planes. We emphasize Spotlight's contribution to these segmentations by coloring blue all contours whose plane was chosen automatically. Fig. 5 (row 1) shows Spotlight aiding the user in 

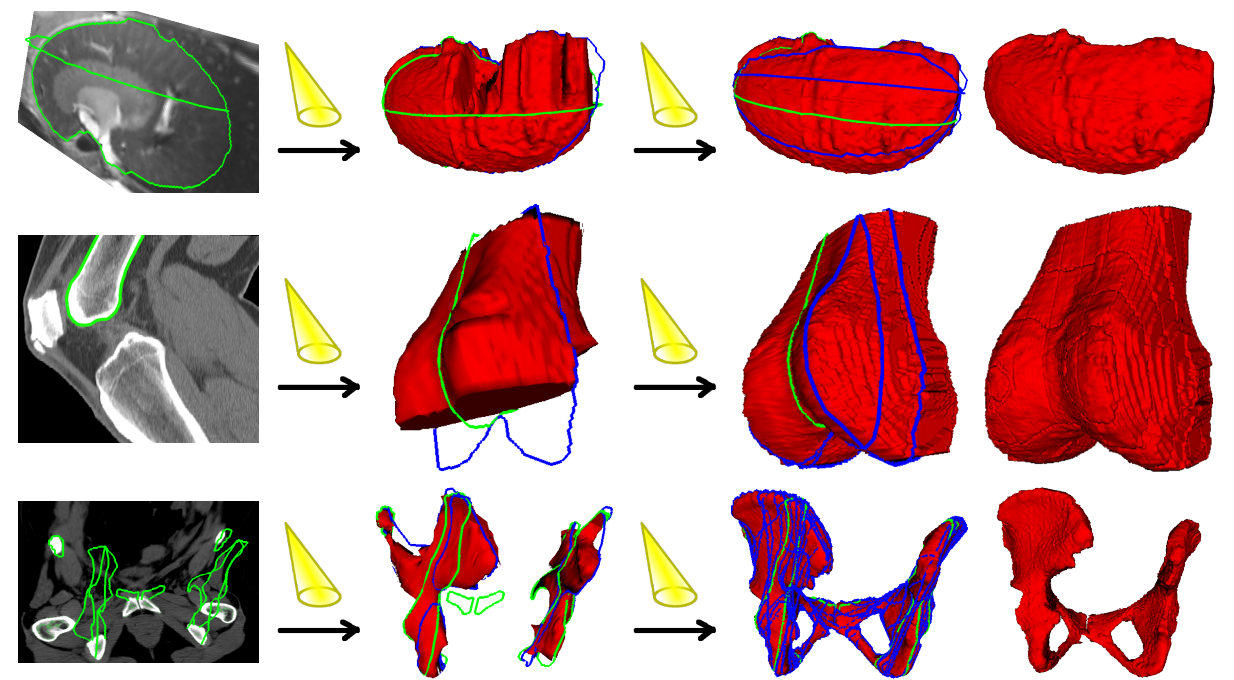

Fig. 5. Segmentation results on real data. (Column 1) Initial contours (green) overlaid on image data slices of (top to bottom) a mouse kidney (MR), femur (CT) and pelvis (CT), respectively. Contours were provided on 2, 1 and 4 different initial planes, respectively. (Column 2) Intermediate result showing some added Spotlight contours (blue) and surface rendering mid-way through the Spotlight suggestion process; after 1, 1, and 3 iterations, respectively. (Column 3) Final surface rendering with contours; 4, 4 and 30 iterations of Spotlight were required, respectively. (Column 4) Surface rendering of final segmentation.

segmenting a mouse kidney in a magnetic resonance (MR) image. The data is noisy and contains weak edges, resulting in low Livewire local path costs and this is detected by Spotlight. Fig. 5 (row 2) shows a femur being segmented in a computed tomography (CT) image. Note that Spotlight selects planes that best outline object features such as the lateral and medial condyle. The CT pelvis results in Fig. 5 (row 3) demonstrate our algorithm's ability to navigate real anatomy with non-spherical topology. While Spotlight at first concentrated on correcting errors in the iliam, eventually segmentation confidence increased in that region and Spotlight began focusing on the smaller ischium region.

As a baseline verification of Spotlight, we have tested it against random plane selection. Fig. 6 shows the progress of a knee femur segmentation (from the same image used in Fig. 5, row 2) guided by random plane suggestions. As expected, Fig. 6 shows significantly poorer random plane selection results compared to Spotlight (Fig. 5, row 2). 

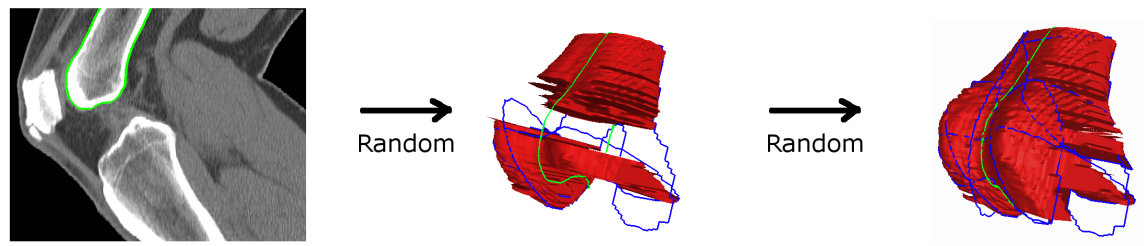

Fig. 6. Segmentation guided by random plane suggestions. Both segmentations were initialized with the same single contour as used in Fig. 5 row 2. The middle image shows the segmentation after 4 randomly chosen contour planes. The right image is after 10 randomly chosen planes. Here, the blue contours are randomly chosen.

\subsection{User Study Tests}

We have performed a user study in order to quantify the efficiency increase provided by Spotlight over the basic T-LW. In the study, 8 users were asked to segment a synthetic open box (Fig. 4 (row 1)) and a liver in a CT image with provided ground truth from the SLIVER07 MICCAI Grand Challenge [4]. For each image, 4 users were instructed to perform the segmentation without using Spotlight, while the other 4 were instructed to use Spotlight. Users were asked to stop segmenting when they deemed the resulting segmentation accurate. The accuracy of the intermediate $3 \mathrm{D}$ segmentation was subsequently evaluated offline after each added contour. The quantitative results of the study are shown in Fig. 7. Fig. 7 shows the increase in Average Dice Similarity coefficient (DSC) vs. number of mouse clicks, number of contours segmented, and time spent. Without Spotlight, the subjects had difficulty determining where to contour next, often choosing to contour planes that did not improve the segmentation at all. The box image was interesting in that there exists a minimum number of contours required for the T-LW algorithm to segment it completely, yet users were typically unable to intuitively determine this best solution. Compared to the original T-LW algorithm in the box image, Spotlight required only $30 \%$ of the time and $64 \%$ of the contours. For the liver image, total segmentation time was reduced by $35 \%$. Notice also that Spotlight is more consistent in improving the segmentation, exhibiting much smaller standard deviation across users in the DSC.

\section{Conclusions}

We presented Spotlight, a novel confidence-based user guidance technique for increasing efficiency of interactive 3D segmentation. Spotlight's intuitive mechanism automatically draws the user's attention to potential 

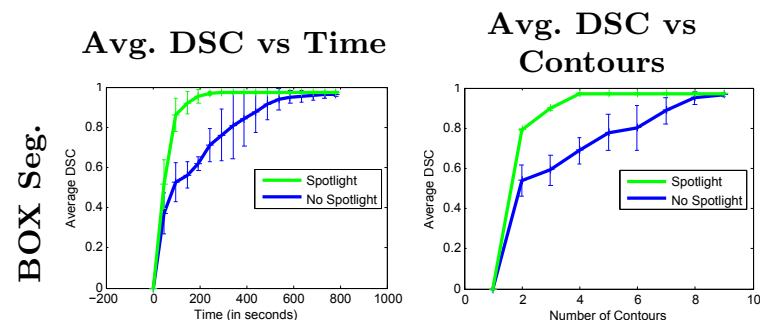

Avg. DSC vs Clicks
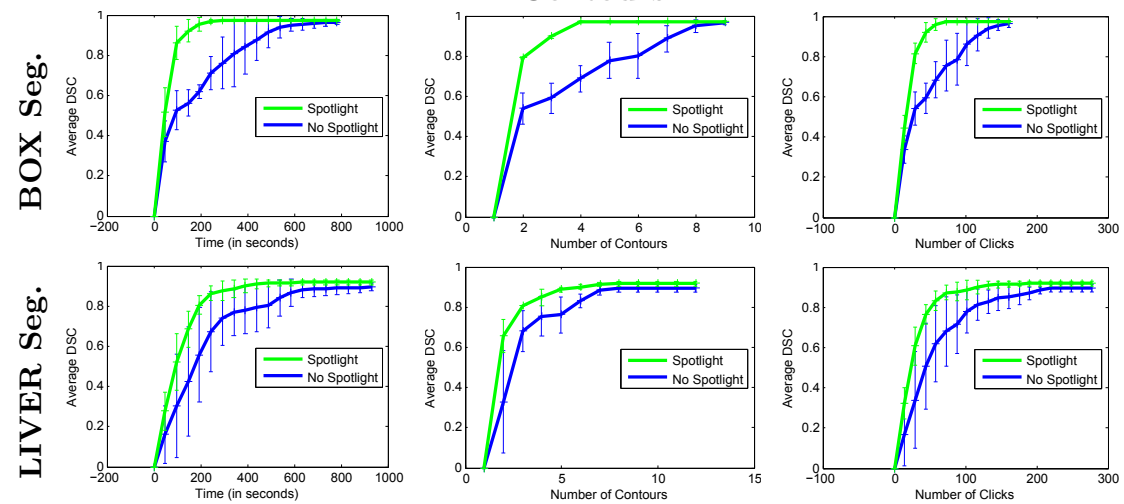

Fig. 7. User study results. Each graph compares Spotlight (green) to the original TLW (No Spotlight, in blue). In the top row, users were asked to segment the synthetic open box shape of Fig. 4 (row 1). In the bottom row, users were asked to segment the liver in a CT image. The first, second and third columns show how the segmentation accuracy improves as a function of time spent, number of contours added, and number of mouse clicks, respectively. The error bars represent the standard deviation among the 4 user results.

problematic regions by highlighting image planes where user input is very likely to improve the segmentation. To quantify confidence in a segmented contour, we minimized an objective function comprising three metrics reflecting contour edge strength, contour stability and object connectivity. We demonstrated Spotlight's successful application for the segmentation of illustrative synthetic volumes and complex real 3D medical images.

Our current implementation was based on the T-LW algorithm [12], which we extended with an analytical formulation for the turtle map. This enabled the necessary support for using arbitrarily directed oblique planes that may be suggested by Spotlight. The Spotlight strategy, however, is not restricted to this Livewire framework and can be incorporated in to other interactive segmentation algorithms, such as random walker with precomputation [7] or graph cuts [9]. We leave these extensions to future work. Our algorithm can also easily support additional confidence measures to augment the current SAs. Part of our future work will focus on incorporating region-based reliability metrics. Furthermore, we are currently investigating the formulation of Spotlight under an active learning framework. Finally, we will focus on further testing of Spotlight in various applications and performing extensive validation experiments on real data through additional user studies. 


\section{References}

1. C. J. Armstrong, B. L. Price, and W. A. Barrett. Interactive segmentation of image volumes with live surface. Computers and Graphics, 31(2):212-229, 2007.

2. W. A. Barrett and E. N. Mortensen. Interactive live-wire boundary extraction. Medical Image Analysis, 1:331-341, 1997.

3. Y. Boykov and G. Funka-Lea. Graph cuts and efficient N-D image segmentation. International Journal of Computer Vision, 70(2):109-131, 2006.

4. T. Heimann et al. Comparison and evaluation of methods for liver segmentation from CT datasets. IEEE Transactions on Medical Imaging, 28(8):1251-1265, 2009.

5. L. Grady. Random walks for image segmentation. IEEE Transactions on Pattern Analysis and Machine Intelligence, 28(11):1768-1783, 2006.

6. L. Grady and G. Funka-Lea. An energy minimization approach to the data driven editing of presegmented images/volumes. In Medical Image Computing and Computer-Assisted Intervention, volume 4191/2006, pages 888-895, 2006.

7. L. Grady and A. K. Sinop. Fast approximate random walker segmentation using eigenvector precomputation. In IEEE Computer Vision and Pattern Recognition, pages 1-8, 2008.

8. Y. Kang, K. Engelke, and W. Kalender. Interactive 3D editing tools for image segmentation. Medical Image Analysis, 8:35-46, 2004.

9. P. Kohli and P. H.S. Torr. Measuring uncertainty in graph cut solutions - efficiently computing min-marginal energies using dynamic graph cuts. In ECCV Computer Vision, pages 30-43, 2006.

10. F. Malmberg, E. Vidholm, and Nyström. A 3D live-wire segmentation method for volume images using haptic interaction. In Discrete Geometry for Computer Imagery, volume 4245/2006, pages 663-673, 2006.

11. S. Olabarriaga and A. Smeulders. Interaction in the segmentation of medical images: a survey. Medical Image Analysis, 5:127-142, 2001.

12. M. Poon, G. Hamarneh, and R. Abugharbieh. Efficient interactive 3D livewire segmentation of complex objects with arbitrary topology. Computerized Medical Imaging and Graphics, 32:639-650, 2008.

13. J. Y. Yen. Finding the K shortest loopless paths in a network. Management Science, 17:712-716, 1971. 